POS $\quad \begin{aligned} & \text { PROCEEDINGS } \\ & \text { OF SCIENCE }\end{aligned}$

\title{
Mass measurements of radionuclides near the endpoint of the rp-process at SHIPTRAP
}

\section{Gleb Vorobjev ${ }^{1,2^{*}}$, Dieter Ackermann ${ }^{1}$, Dietrich Beck ${ }^{1}$, Klaus Blaum ${ }^{1,3}$, Michael Block $^{1}$, Ankur Chaudhuri ${ }^{4}$, Zhenyu $\mathrm{Di}^{5}$, Sergey Eliseev ${ }^{1,2}$, Rafael Ferrer ${ }^{3}$, Dieter Habs $^{6}$, Frank Herfurth ${ }^{1}$, Fritz Hessberger ${ }^{1}$, Sigurd Hofmann ${ }^{1}$, H.-Jürgen Kluge ${ }^{1,7}$, Giancarlo Maero ${ }^{1}$, Ana Martín ${ }^{1}$, Gerrit Marx ${ }^{4}$, Marco Mazzocco ${ }^{1}$, Jürgen B. Neumayr $^{6}$, Yuri Novikov ${ }^{2}$, Wolfgang Plass ${ }^{5}$, Christian Rauth $^{1}$, Daniel Rodríguez ${ }^{8}$, Christoph Scheidenberger ${ }^{1,5}$, Lutz Schweikhard ${ }^{4}$, Michael Sewtz $^{6}$, Peter Thirolf ${ }^{6}$, Wolfgang Quint ${ }^{1}$, and Christine Weber ${ }^{1}$.}

\footnotetext{
${ }^{1}$ Gesellschaft für Schwerionenforschung (GSI), 64291 Darmstadt, Germany.

${ }^{2}$ Sankt-Petersburg Nuclear Physics Institute, 188300 Gatchina, Russia.

${ }^{3}$ Johannes-Gutenberg-Universität, 55099 Mainz, Germany

${ }^{4}$ Ernst-Moritz-Arndt-Universität, 17489 Greifswald, Germany.

${ }^{5}$ Justus-Liebeg-Universität, 35392 Giessen, Germany.

${ }^{6}$ Ludwig-Maximilians-Universität 85748 Garching, Germany.

${ }^{7}$ Ruprecht-Karls-Universität 69120 Heidelberg, Germany.

${ }^{8}$ LPC, ENSICAEN, 14050 Caen Cedex, France.
}

\begin{abstract}
The Penning trap mass spectrometer SHIPTRAP at GSI Darmstadt allows high-accuracy mass spectrometry of fusion-evaporation residues, produced and separated at the velocity filter SHIP. The production technique gives access to nuclei far off the beta stability line. To match the energy of the products to the energy acceptance of the trap a buffer gas stopping cell is used. In the experiment described here, $\mathrm{Sn}, \mathrm{In}, \mathrm{Cd}$ and $\mathrm{Ag}$ isotopes were produced in the reaction ${ }^{50} \mathrm{Cr}+$ ${ }^{58} \mathrm{Ni}$ and the masses of 13 nuclides could be measured with an accuracy better than $10^{-7}$. Our results agree well with the data derived from $\beta^{+}$-decay studies but allow for a more accurate determination of the proton separation energies and $\mathrm{Q}_{\beta}$-values. This is important to improve reaction network calculation of the rp-process.
\end{abstract}

International Symposium on Nuclear Astrophysics - Nuclei in the Cosmos - IX

CERN, Geneva, Switzerland

25-30 June, 2006

\footnotetext{
*Speaker E-mail: g.vorobjev@gsi.de 


\section{Introduction}

The nuclides with mass numbers $A$ between 80 and 100 and equal numbers of protons and neutrons $N=Z$ are subject of special interest because different facets of nuclear physics appear in this region. The $N=Z$ line crosses here the limit of stability against proton emission. The neutron and proton shell closure at $N=Z=50$ and in particular the doubly magic nucleus ${ }^{100} \mathrm{Sn}$ are prime applications for the shell model calculations. The nuclear shape changes rapidly in this region and gives rise to long-lived isomers with small excitation energy.

Apart from these nuclear physics aspects, the knowledge of the nuclear properties in this region is of importance for nuclear astrophysics because the rapid proton (rp) capture process is passing just through this area of the chart of nuclides [1]. This process, which is in part responsible for the synthesis of the elements, proceeds during star bursts along the $N=Z$ line up to ${ }^{56} \mathrm{Ni}$. For higher $Z$ values, proton capture rates become smaller and the competing photodisintegration becomes stronger. This makes the reaction path broader and shifts it from the $N=Z$ line towards the beta stability by one or two mass units (Fig. 1). This displacement can be even stronger for steady state burning conditions as for instance in X-ray pulsars [2].

The actual path of the rp process is defined by the conditions in the stellar environment and by the properties of the nuclei involved. Mass differences, i.e. proton separation energies and $\beta^{+}$-decay Q-values together with beta-decay half-lives are needed to perform rp-process network calculations. Additionally, spectroscopic data are required in order to calculate the abundance of nuclides in the cooling stage after the burst. The presence of long-lived isomeric states at the rp-process path is also important because these states have to be included in the calculations and might affect both the pathway as well as the timescale of the process.

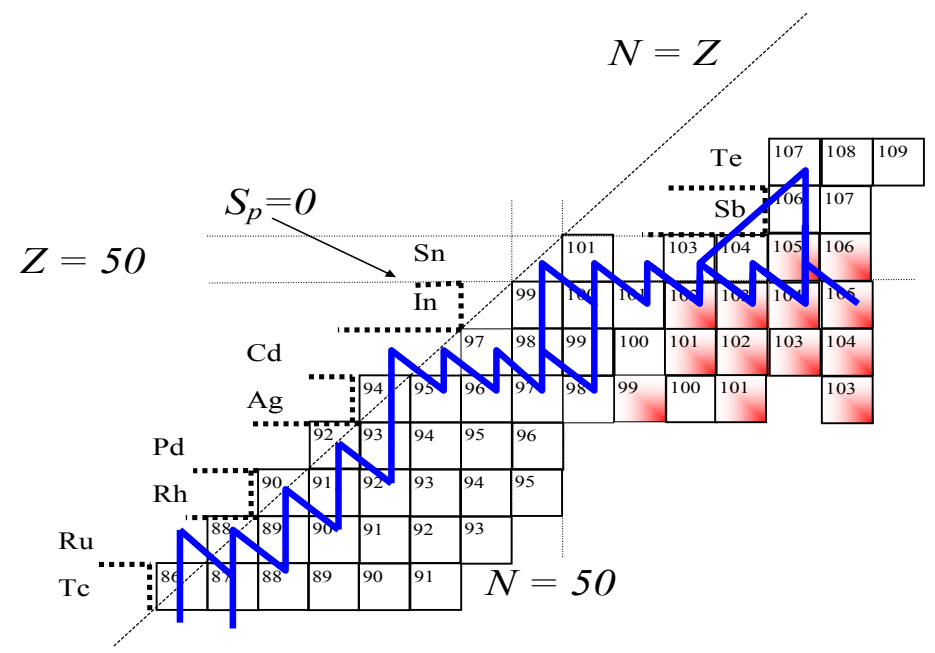

Figure 1. Part of the chart of nuclides. The blue line indicates a probable rp-process path [1]. Nuclides marked in red were measured at SHIPTRAP in the present work.
Unfortunately, most masses of nuclides along the rp-process pathway have not been determined experimentally. Due to this lack of knowledge, the input data for network calculations are presently based mainly on model predictions. Generally, these predictions by various models deviate strongly from each other. Therefore, it is very important to perform direct mass measurements. Even if the nuclei of direct interest cannot be accessed, it will be useful to study nuclei in their vicinity, because then the predictive power of mass models can be improved. This short note 
describes an experiment at SHIPTRAP in which a ${ }^{50} \mathrm{Cr}$ beam with an energy of $3.6 \mathrm{MeV} / \mathrm{u}$ was used to irradiate ${ }^{58} \mathrm{Ni}$ target to produce the nuclei of interest and measure their mass.

\section{Experimental set-up}

The ion trap facility SHIPTRAP [3] at GSI in Darmstadt was set up to enable precision experiments on heavy elements produced in fusion-evaporation reactions and separated by the velocity filter SHIP [4]. A schematic drawing of the setup is shown in Fig. 2. The reaction products from SHIP with energies of a few $100 \mathrm{keV} / \mathrm{u}$ are stopped in a stopping cell filled with buffer gas [5]. To obtain high efficiency and to improve the ion beam quality for an efficient injection into the Penning trap, the ion beam extracted from the stopping cell passes through the Extraction and Buncher radio-frequency quadrupoles (RFQ). In these buffer-gas-filled four-rod

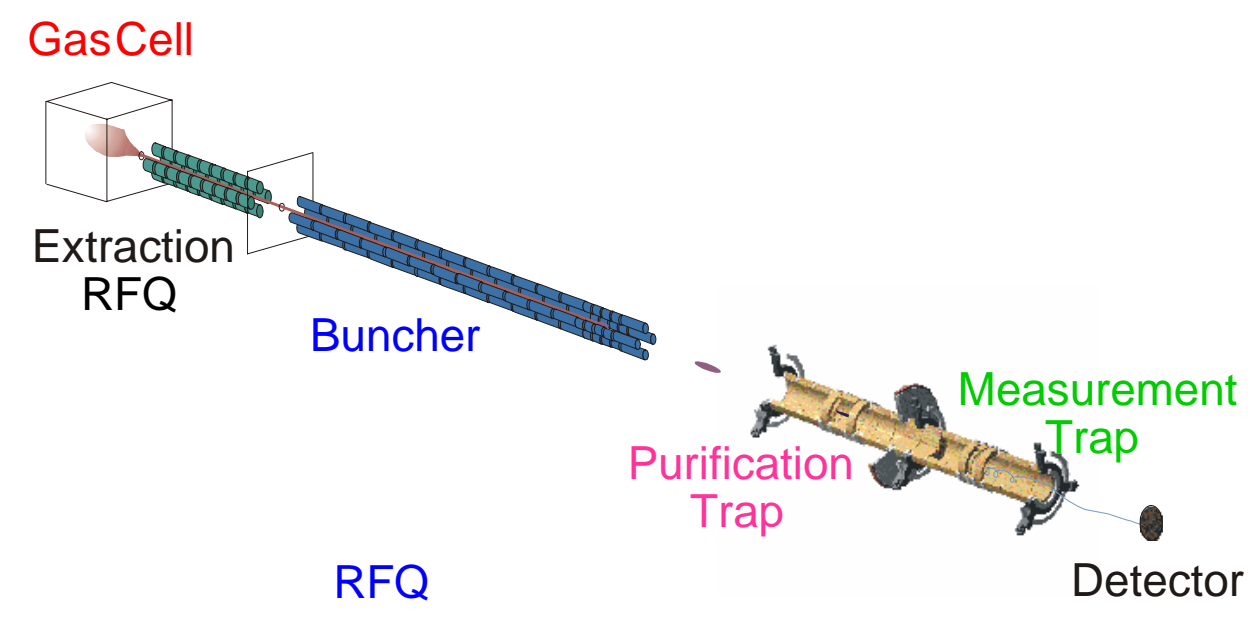

Figure 2. Schematic layout of the SHIPTRAP facility.

structures the ions are cooled within a few milliseconds, accumulated in the Buncher RFQ, and extracted as a low-emittance bunched beam. Then the ions are transported into the Penning trap system. It consists of two cylindrical Penning traps in a common superconducting magnet of $7 \mathrm{~T}$ field strength. The first trap, with a mass resolving power of about 100000 at mass numbers $A \approx$ 100 , is used for isobaric purification. In the second trap mass measurements are performed. For this. the cyclotron frequency

$$
v_{c}=\frac{1}{2 \pi} \frac{q B}{m_{\text {ion }}},
$$

is measured for an ion with charge $q$ and unknown mass $m_{i o n}$ in a magnetic field of magnitude $B$ by the time-of-flight ion cyclotron resonance method [6,7]. An example of such a measurement is shown in Fig. 3. To determine an atomic mass from the cyclotron frequency the magnitude of the magnetic field has to be known very precisely. This is achieved by a linear interpolation of the cyclotron frequencies $v_{\text {ref }}$ of a stable isotope with well known mass measured before and after the determination of the cyclotron frequency of the ion of interest. From the ratio of these two frequencies the atomic mass $m$ of the nuclide of interest can be obtained by 


$$
m_{\text {atomic }}=\frac{q}{q_{r e f}}\left(m_{r e f}-q_{r e f} \cdot m_{e}\right) \frac{v_{c, r e f}}{v_{c}}+q \cdot m_{e},
$$

using the mass $m_{r e f}$ and charge state $q_{r e f}$ of the reference ion and the electron mass $m_{e}$, where the atomic binding energy is assumed to be negligible. In additional to the statistical uncertainties,

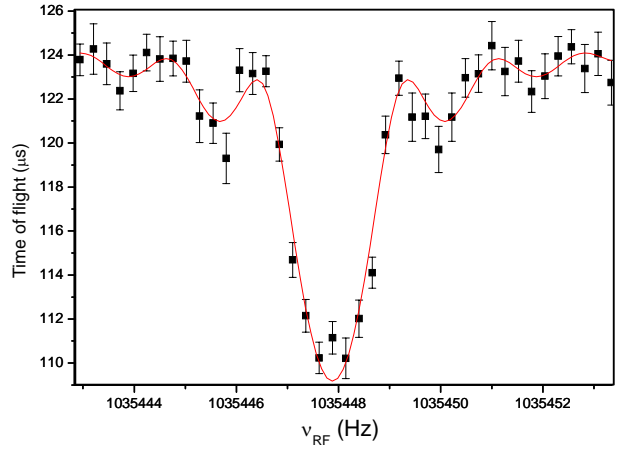

Figure 3. Cyclotron resonance (TOF versus excitation frequency) of ${ }^{104} \mathrm{Cd}$. The red line is a fit of the theoretical line shape to the data points.

non-linear changes of the magnetic field, systematic uncertainties due to the mass difference of reference ion and ion of interest and the residual systematic uncertainty of the apparatus determine the achievable precision of a measurement $[6,8]$. Relative mass uncertainties of about $5 \cdot 10^{-8}$ are routinely obtained.

Presently, SHIPTRAP is able to measure masses with production cross sections down to 100 $\mu$ barn and to resolve isomers with excitation energies of about $100 \mathrm{keV}$ at mass number $A \approx$ 100. The overall efficiency of SHIPTRAP is currently about $0.5 \%$. It is mainly limited by the total efficiency of the gas cell of $5-8 \%$ under optimal conditions. The transmission efficiency of the cooler RFQ was determined to be $50 \%$ and the efficiency of the micro channel plate detectors (MCP) is $15-30 \%$. Those values were measured by stopping $\alpha$-radioactive recoil ions inside the gas-cell and by counting them with a $\mathrm{Si}(\mathrm{Au})$ detector after extraction [9].

\section{Results}

A ${ }^{50} \mathrm{Cr}^{11+}$ beam with $3.6 \mathrm{MeV} / \mathrm{u}$ energy and an average intensity of $40 \mathrm{pnA}$ was used to irradiate a ${ }^{58} \mathrm{Ni}$ target wheel $0.5 \mathrm{mg} / \mathrm{cm}^{2}$. Red squares in Fig. 1 indicate the nuclides which are produced by evaporation of few protons and neutrons from the compound nuclei ${ }^{108} \mathrm{Te}$. As an example, ${ }^{102}$ In is produced via the $3 \mathrm{n} 3 \mathrm{p}$ evaporation channel with a calculated cross section of 0.6 mbarn. If this is compared to the ions detected at the MCP, an overall efficiency of about 1\%o can be deduced. Preliminary results of the mass measurements are presented in Table 1. The final determination of the systematic mass uncertainty and a careful treatment of possible isomeric admixtures are still pending.

In Table 1, the masses determined in this work are compared with those from literature [10]. Except for ${ }^{105}$ In, the measured mass excesses agree with the values of the AME-2003 quite well within the error bars. However, the difference between the present values and the literature values shows a certain trend for the indium isotopes. The absolute value of the mass excess difference seems to increase systematically with increasing neutron number.

As was discussed in reference [2], the rp-process proceeds along the tin isotopic chain until the proton separation energy of the antimony isotopes becomes large enough to delay photo-disintegration and, hence, causes the rp-process to proceed to heavier elements. This happens around ${ }^{105} \mathrm{Sn}$, but the current uncertainties in the proton separation energies of 
antimony isotopes prevented an accurate prediction which of the various $\mathrm{SnSbTe}$ cycles dominates. Now, by using the mass values measured in this work, more accurate proton separation energies can be deduced in order to clarify the situation.

Table 1. Preliminary results of the mass measurements performed at SHIPTRAP. The frequency ratio $r$ of the measured nuclide is given with respect to the frequency of ${ }^{85} \mathrm{Rb}^{+}$used as reference mass. The resulting mass excess $\left(\mathrm{ME}=\mathrm{m}_{\mathrm{atom}}-\mathrm{A} \cdot \mathrm{u}\right)$ is given and compared to the values given in AME-2003, the Atomic Mass Evaluation performed in 2003 [10].

\begin{tabular}{|c|c|c|c|}
\hline & Frequency ratio & $\mathrm{ME}_{\text {present work }}(\mathrm{keV})$ & $\mathrm{ME}_{\text {present work }}-\mathrm{ME}_{\text {AME }}(\mathrm{keV})$ \\
\hline${ }^{99} \mathrm{Ag}$ & $1.164946903(67)$ & $-76721.6(5.3)$ & $38(150)$ \\
\hline${ }^{101} \mathrm{Ag}$ & $1.188442505(85)$ & $-81341.1(6.7)$ & $-121(100)$ \\
\hline${ }^{103} \mathrm{Ag}$ & $1.211952784(100)$ & $-84799.8(8.0)$ & $-9(17)$ \\
\hline${ }^{101} \mathrm{Cd}$ & $1.188511951(77)$ & $-75848.3(6.1)$ & $-98(150)$ \\
\hline${ }^{102} \mathrm{Cd}$ & $1.200240628(69)$ & $-79670.7(5.5)$ & $7(29)$ \\
\hline${ }^{103} \mathrm{Cd}$ & $1.212005234(75)$ & $-80651.3(5.9)$ & $-2(15)$ \\
\hline${ }^{104} \mathrm{Cd}$ & $1.223740159(64)$ & $-83979.5(5.1)$ & $-5(9)$ \\
\hline${ }^{102} \mathrm{In}$ & $1.200354128(66)$ & $-70693.3(6.6)$ & $17(110)$ \\
\hline${ }^{103} \mathrm{In}$ & $1.212081280(70)$ & $-74636.5(5.5)$ & $-38(25)$ \\
\hline${ }^{104} \mathrm{In}$ & $1.223838895(63)$ & $-76170.0(5.0)$ & $-60(80)$ \\
\hline${ }^{105} \mathrm{In}$ & $1.235572018(72)$ & $-79640.7(5.7)$ & $-160(80)$ \\
\hline${ }^{105} \mathrm{Sn}$ & $1.235651663(72)$ & $-73341.3(5.7)$ & $-81(80)$ \\
\hline${ }^{106} \mathrm{Sn}$ & $1.247377978(76)$ & $-77350.4(6.1)$ & $80(50)$ \\
\hline
\end{tabular}

\section{References}

[1] H. Schatz et al., Physics Reports 294, 167 (1998).

[2] H. Schatz et al., Nuclear Physics A 688, 150c (2001).

[3] J. Dilling et al., Hyperfine Interact. 127, 491 (2000).

[4] S. Hofmann, G. Münzenberg, Rev. Mod. Phys. 72, 733 (2000).

[5] J. B. Neumayr et al., Nucl. Instr. Meth. B 244, 489 (2006).

[6] K. Blaum, Physics Reports, 425, 1 (2006).

[7] G. Gräff, H. Kalinowski, J. Traut, Z. Phys. A 297, 35 (1980).

[8] A. Kellerbauer et al., Eur. Phys. J. D 22, 53 (2003).

[9] M. Block et al., Eur. Phys. J. A 25, 49 (2005).

[10] G.Audi, A.H.Wapstra and C.Thibault, Nucl. Phys. A 729, 337 (2003). 